\title{
CEACAM6 Positive
}

National Cancer Institute

\section{Source}

National Cancer Institute. CEACAM6 Positive. NCI Thesaurus. Code C159905.

An indication that CEACAM6 expression has been detected in a sample. 